\title{
Mcl-1 expression is up-regulated in malignancies of the parotid gland
}

\author{
Gregor Heiduschka ${ }^{\mathrm{a}}$, Boban M. Erovic ${ }^{\mathrm{a}}$, Johannes Pammer ${ }^{\mathrm{b}}$, Ulana Kotowski ${ }^{\mathrm{a}}$, Alexandra Kaider ${ }^{\mathrm{c}}$, \\ Matthaeus Ch. Grasl ${ }^{\mathrm{a}}$ and Dietmar Thurnher ${ }^{\mathrm{a}, *}$ \\ ${ }^{a}$ Department of Otorhinolaryngology, Head and Neck Surgery, Comprehensive Cancer Center, Medical University \\ of Vienna, Austria \\ ${ }^{\mathrm{b}}$ Department of Otorhinolaryngology, Pathology, Comprehensive Cancer Center, Medical University of Vienna, \\ Austria \\ ${ }^{\mathrm{c}}$ Department of Otorhinolaryngology, Medical Computer Sciences, Comprehensive Cancer Center, Medical \\ University of Vienna, Austria
}

\begin{abstract}
The anti-apoptotic protein Mcl-1 is highly expressed in various types of malignant tumors. Overexpression is reported to correlate with poor prognosis and disease progression. We report the expression levels of Mcl-1 in tumor samples of the parotid gland. A retrospective study containing 108 patients was performed. A tissue microarray of six malignancies of the parotid gland and pleomorphic adenoma as control was constructed. Parotid gland tumor samples were immunohistochemically stained for Mcl-1 and expression intensities were assessed. Statistical analysis included correlation to patients' clinical data and comparison of malignancies to the adenoma.

All malignancies had significantly higher expression of Mcl-1 than the pleomorphic adenomas. The intensity, however, had no significant correlation to overall survival.

Our immunohistochemical findings indicate that parotid gland malignancies produce high levels of Mcl-1 protein. Therefore, Mcl-1 might serve as a predictive co-marker in tumors of the parotid gland.
\end{abstract}

Keywords: Mcl-1, cancer, parotid, salivary gland, head and neck

\section{Introduction}

Malignant tumors of the salivary glands are rare and account for $3-10 \%$ of all head and neck tumors $[1,2]$. The incidence is estimated with 0.6 per 100,000 population per year [3]. The vast majority of salivary gland lesions occur in major salivary glands, less common sites include the minor salivary glands of the tongue, floor of mouth, lips and palate [2]. Within the major salivary glands, the parotid gland is involved in up to 85 percent [4]. Morphologic diversity is a hallmark of these tumors. The current WHO classification differ-

\footnotetext{
*Corresponding author: Dietmar Thurnher, MD, Department of Otorhinolaryngology, Head and Neck Surgery, Medical University of Vienna, Waehringer Guertel 18-20, A-1090 Vienna, Austria. Tel.: +43 140400 3372; Fax: +43 140400 3355; E-mail: dietmar.thurnher @meduniwien.ac.at.
}

entiates 13 benign and 24 malignant entities, excluding the subtypes [5].

Histological type, size of tumor at time of diagnosis, advanced age as well as local invasion determine the survival of patients with malignant tumors of the parotid gland [4,6]. So far there are only a few biological markers that allow reliable prediction of survival $[7,8]$.

Tumor growth depends on excessive proliferation and insufficient regulation of the apoptotic signal transduction. The B-cell lymphoma 2 (Bcl-2) proteins were found to be key elements in regulating apoptosis after a cell is exposed to cytotoxic agents or in response to physiological cues. The Bcl-2 family comprises both pro-apoptotic proteins like Bax, Bak or Bad and antiapoptotic proteins like Bcl-2, Bcl-XL and Mcl-1. A disbalance of these two groups influences the susceptibility of cells to apoptosis through cytochrome $c$ release [9]. 
The antiapoptotic myeloid cell leukemia (Mcl-1) protein is strongly expressed in various malignant tissues such as cancers of the colon [10], head and neck [11], or the liver [12]. Mcl-1 counteracts proapoptotic proteins including Noxa, Puma and Bim and thus inhibits their induction of cytochrome $c$ release [9]. Overexpression of Mcl-1 is associated with disease progression, resistance to chemotherapy, and poor clinical outcome. Furthermore, Mcl-1 was suggested to be a prognostic marker in gastric cancer [13] and clear-cell renal cancer [14]. In parotid glands, the Bcl-2 protein was already studied and a correlation between high levels of Bcl-2 and low survival was assessed [15].

In the present study we evaluated the expression of the anti-apoptotic protein Mcl-1 in 108 tumors of the parotid gland. We compared malignant tumors to pleomorphic adenomas and correlated expression intensity to clinical data in order to assess whether Mcl-1 may serve as a molecular predictive and prognostic marker.

\section{Materials and methods}

\subsection{Patients}

108 archival samples of tumors of the parotid gland from the Department of Otorhinolaryngology, Head and Neck Surgery were included in this study. All patients had undergone surgery between 1970 and 2004. The age range was from 17.8 to 90.2 years (median 61.7 years). Fifty patients $(46.3 \%)$ were females and $58(53.7 \%)$ males. Median follow up was 76.9 months.

\subsection{Tissue Micro Array construction}

Initially, $5 \mu \mathrm{m}$ sections from each tissue block were stained with haematoxylin-eosin to check for the presence of cancer tissue. Thus, our pathologist (J.P.) marked morphologically representative areas and sampled $0.6 \mathrm{~mm}$ tissue cores using a punch (Beecher Instruments, Sun Prairie, WI, USA). Samples consisted of three malignant cores from different sites of the tumor per patient.

\subsection{Immunohistochemistry}

Dewaxed and rehydrated tumor sections (of 2-3 $\mu \mathrm{m}$ thickness) were subjected to antigen retrieval in a microwave oven (600W) employing 1 mM EDTA, pH 8.0, buffer. Blocking of unspecific binding was achieved with 5\% TBS/BSA (Sigma-Aldrich, Germany) for 1 $\mathrm{h}$ at room temperature (RT). Application of the mouse monoclonal Mcl-1 antibody (Novocastra, Laboratories, Newcastle upon Tyne, UK) was done at $4{ }^{\circ} \mathrm{C}$ overnight. A secondary biotinylated antibody (1:200, Multilink; Dako, Denmark) in $1 \%$ TBS/BSA was added for 1 $\mathrm{h}$ at RT, followed by alkaline phosphatase conjugated Streptavidin-AP/TBS/BSA (1:250, Dako, Glostrup, Denmark) for $1 \mathrm{~h}$ at RT. Visualisation was performed with fast red (Sigma, Missouri, USA) and counterstained with haemalaun. Samples were analyzed using an Olympus BH-2 microscope.

\subsection{Specimen classification based on immunohistochemical results and morphological features}

All samples were assigned to one of four categories of marker expression (none): < 5\%; 1 (weak): 5-30\%; 2 (moderate): $30-60 \% ; 3$ (strong): $60-100 \%$. Cut-off points were used as previously described [16]. The expression pattern was determined by three investigators (G.H., B.E., J.P.) without knowledge of patients' clinical data. Expression score was defined as average of the expression intensities in each tumor group.

\subsection{Statistical analysis}

To compare the Mcl-1 expression intensities between the seven tumor entities the nonparametric KruskalWallis test was used. Pairwise comparisons were performed between the six malignant tumor groups and the pleomorphic adenomas using the Wilcoxon rank sum test. Due to the rather small sample sizes exact p-values were calculated using the program StatXact (Cytel Studio Version 8.0, Cytel Inc. 2007). The Bonferroni-Holm correction was applied to adjust the p-values for the number of multiple comparisons performed. Survival probabilities were estimated by the Kaplan-Meier method. To evaluate the unadjusted and tumor-type-adjusted effect of Mcl-1 expression on survival univariate and multiple Cox-Regression models were performed.

\section{Results}

\subsection{Clinical data}

All patients had a tumor in the parotid gland and the tumor entities are listed in Table 1. Overall survival is shown in Fig. 1. Squamous cell carcinoma patients had the shortest median overall survival of 11.7 months, whereas adenoid cystic carcinoma patients had a median overall survival of 73.4 months. 
Table 1

Patient data and mcl-1 expression

\begin{tabular}{lcccc}
\hline histological type & patient N & $\begin{array}{c}\text { median survival } \\
\text { (months) }\end{array}$ & $\begin{array}{c}\text { expression } \\
\text { score }\end{array}$ & p-value \\
\hline adenocarcinoma & 17 & 35.4 & 1.44 & 0.013 \\
adenoidcystic carcinoma & 14 & 73.4 & 1.69 & 0.017 \\
acinic cell carcinoma & 6 & 63.7 & 2.00 & 0.017 \\
mucoepidermoid carcinoma & 12 & 66.2 & 1.83 & 0.007 \\
squamous cell carcinoma & 17 & 11.7 & 2.21 & 0.0001 \\
carcinoma ex pleomorphic adenoma & 11 & 56.3 & 1.78 & 0.017 \\
pleomorphic adenoma & 31 & & 0.67 & \\
loss of tissue & 10 & & & \\
\hline
\end{tabular}

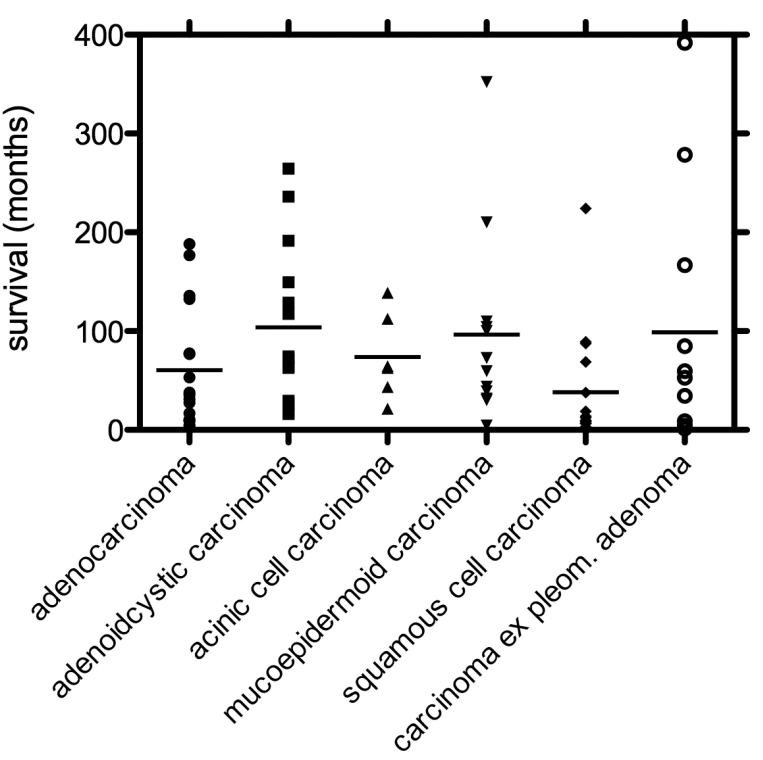

Fig. 1. Survival in months shown for each malignant tumor entity of the parotid gland. The line indicates the median overall survival.

\subsection{Immunohistochemistry}

A tissue microarray containing tumor samples of 108 patients was stained with Mcl-1 antibody by immunohistochemistry. During the staining process, we observed a loss of tissue in $10(9.3 \%)$ out of 108 samples. Expression scores of all tumor groups are shown in Table 1.

\subsubsection{Adenocarcinoma}

One sample $(5.9 \%)$ out of 17 showed no expression, $8(47.1 \%)$ patients had weak expression and in $6(35.2 \%)$ patients we detected moderate expression. One $(5.9 \%)$ specimen showed strong expression of Mcl-1 and one (5.9\%) tissue was lost. Compared to the control group, Mcl-1 expression was significantly stronger ( $p=0.013)$ (Fig. 2a).

\subsubsection{Adenoid cystic carcinoma}

Two samples (14.3\%) out of 14 showed no expression of Mcl-1. Three biopsies (21.4\%) showed weak expression, 5 tumors $(35.7 \%)$ demonstrated moderate expression and in three patients $(21.5 \%)$ we observed strong expression. One $(7.1 \%)$ sample was loss of tissue. Mcl-1 expression was significantly up regulated compared to pleomorphic adenoma $(p=0.017)$ (Fig. 2b).

\subsubsection{Acinic cell carcinoma}

One $(16.7 \%)$ out of six tumor samples showed weak, two $(33.3 \%)$ samples showed moderate and one (16.7\%) sample showed strong expression. Two samples $(33.3 \%)$ were loss of tissue. Up regulation was significant ( $p=0.017$ ) (Fig. 2c).

\subsubsection{Mucoepidermoid carcinoma}

One $(8.3 \%)$ out of 12 patients was negative for Mcl1 and three $(25 \%)$ samples had only weak expression of Mcl-1. In $5(41.7 \%)$ tumor samples, we detected a moderate and in three $(25 \%)$ we observed a strong expression of Mcl-1. Mcl-1 was significantly over expressed in comparison to the control group $(p=0.007)$ (Fig. 2d).

\subsubsection{Squamous cell carcinoma}

Three (17.6\%) out of 17 tumor samples showed weak expression, $5(29.4 \%)$ biopsies showed moderate expression and $6(35.4 \%)$ specimens showed strong expression of Mcl-1. Three samples (17.6\%) were loss of tissue. Compared to the pleomorphic adenoma, we detected a significantly stronger expression of Mcl-1 in squamous cell carcinoma ( $p<0.0001)$ (Fig. 2e).

\subsubsection{Carcinoma ex pleomorphic carcinoma}

Four (36.4\%) out of 11 biopsies showed weak expression, three (27.2) samples showed moderate expression and two (18.2\%) showed strong expression of Mcl-1. Two samples (18.2\%) were loss of tissue. Staining of Mcl-1 was significantly stronger than in the control group ( $p=0.017$ ) (Fig. 2f). 

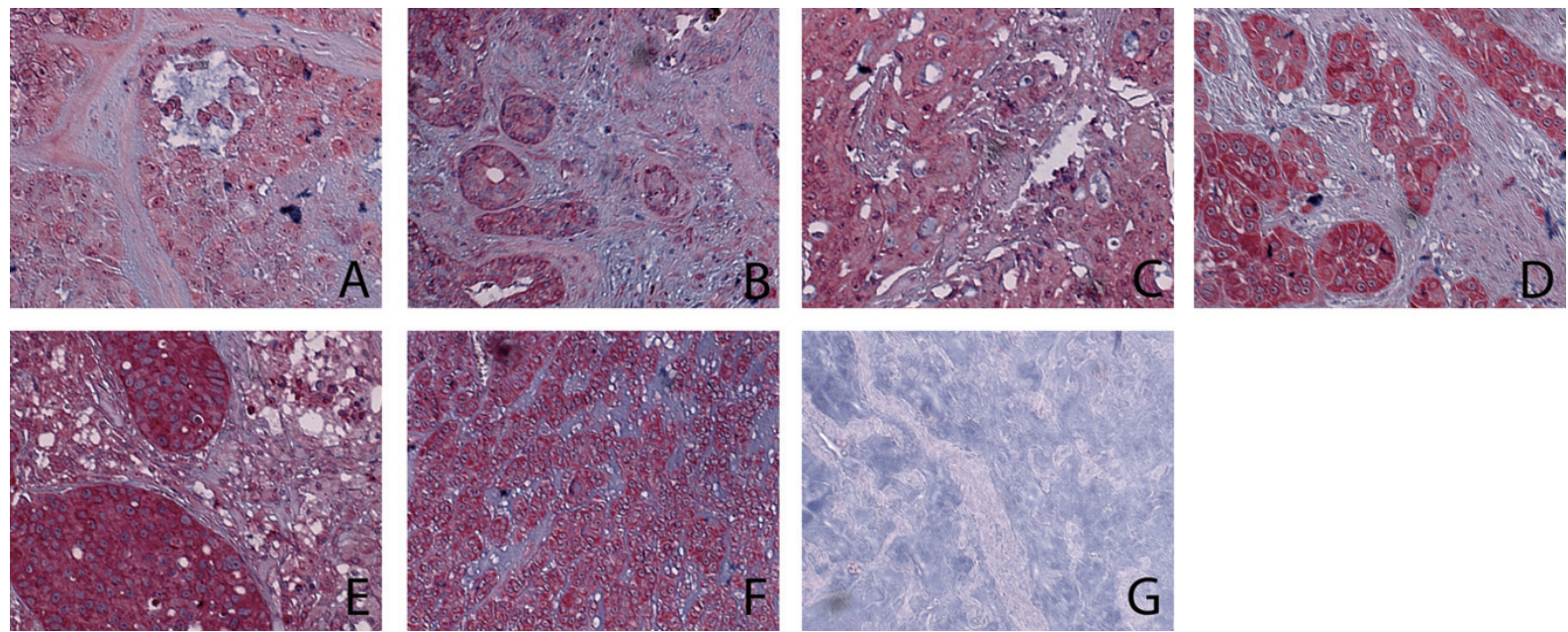

Fig. 2. The immunostaining of Mcl-1 molecules in a tissue microarray of various benign and malignant tumors of the parotid gland. Strong expression of Mcl-1 was shown in adenocarcinoma (a), adenoid cystic carcinoma (b) acinic cell carcinoma (c), mucoepidermoid carcinoma (d), squamous cell carcinoma (e) and carcinoma ex pleomorphic adenoma (f). Pleomorphic adenoma served as control group (g) and showed only weak expression of Mcl-1. All photomicrographs were taken at a magnification x 200 .

\subsubsection{Pleomorphic adenoma}

Eight $(25.8 \%)$ out of 31 tumor samples were negative for Mcl-1, 18 (58.1\%) samples demonstrated weak expression and 4 (12.9\%) biopsies showed moderate expression. One sample (3.2\%) was loss of tissue (Fig. 2g).

\subsection{Correlation of Mcl-1 expression and clinical data}

Statistical analysis revealed no correlation between Mcl-1 expression and overall survival in cancer patients. However, strongest expression was observed in squamous cell carcinoma samples (Table 1).

\section{Discussion}

Dysregulation of apoptosis-regulating proteins plays an important role in the progression of human malignancies. The Bcl-2 family proteins represent one of the biologically relevant classes of apoptosis-regulating gene products. Mcl-1, a member of the Bcl-2 family and $\mathrm{Bcl}-2$ itself, function as antagonists to apoptosis, whereas other members like Bax, Bak, Noxa, Puma or Bim induce cell death. The fragile homeostasis between these two groups determines the survival or death of a cell.

Advanced parotid gland cancer still carries a poor prognosis despite current treatment modalities, including surgery, radiation therapy with or without chemotherapy. Furthermore, a comorbidity of these regimens might be facial nerve palsy, which carries a substantial loss in quality of life for the patient.

The improved knowledge of tumor biology led to the approach of targeted therapy. Tailor-made therapy for every particular tumor is the goal to achieve by identifying molecular targets and designing adequate agents to be used in combination with current chemoradiation treatments. The Bcl-2 antisense oligonucleotide oblimersen in combination with chemotherapy went through a phase III clinical study of melanoma and improved multiple clinical outcomes, however not survival [17]. Another agent is obatoclax, which is a pan$\mathrm{Bcl}$ inhibitor, is currently in preliminary clinical trials in patients with multiple myeloma [18]. As for Mcl-1, in vitro studies and an in vivo study in a mouse model successfully used antisense technology to antagonize Mcl-1 in basal cell carcinoma [19], human sarcoma [20] and squamous cell carcinoma of the head and neck [21]. Our data suggest, that Mcl-1 might qualify for targeted therapy in malignancies of the parotid gland, however depending on the Mcl-1 status of the tumor.

In situations where only a limited amount of tumor material is available, a molecular marker might turn an inconclusive into a positive diagnosis. Ki-67 expression proved to be a prognosticator in salivary gland cancer [22]. Furthermore, the antibody against Ki-67, MIB-1, was identified as a possible prognostic marker in fine needle aspiration for papillary thyroid cancer [23]. Lee et al. described a diagnostic magnetic resonance sensor that uses targeted magnetic nano par- 
ticles to detect cancer cells. They detected as few as 2 cancer cells in $1 \mu \mathrm{l}$ samples from a fine needle aspirate using a technology, which is able to discriminate malignant from nonmalignant cells via differential expression of molecular markers [24]. In the current study we could demonstrate a statistically significant difference in the expression of the antiapoptotic protein Mcl-1 in benign pleomorphic adenoma compared to six different types of parotid malignancies.

However, our statistical analysis showed no significant correlation of Mcl-1 expression with overall survival in the malignancies. We noticed though, that the tumor entity with shortest overall survival, the squamous cell carcinoma, had the strongest expression of Mcl-1.

The lack of correlation between Mcl-1 expression and overall survival of patients with different malignant tumors of the parotid gland suggests that many proapoptotic and anti-apoptotic factors affect the control of human cell death.

In this study we show for the first time the expression intensity in various types of malignant tumors of the parotid gland. Compared with the control group of pleomorphic adenomas, each tumor entity tested had significantly higher expression of Mcl-1. However, the lack of correlation between Mcl-1 expression and overall survival suggests that Mcl-1 cannot be used as a singular prognostic factor in parotid gland tumors.

\section{References}

[1] V.L. Vander Poorten, A.J. Balm, F.J. Hilgers et al., The development of a prognostic score for patients with parotid carcinoma, Cancer 85, 2057-2067.

[2] A.V. Jones, G.T. Craig, P.M. Speight et al., The range and demographics of salivary gland tumours diagnosed in a UK population, Oral Oncol 44, 407-417.

[3] J.P. Klussmann, T. Ponert, R.P. Mueller et al., Patterns of lymph node spread and its influence on outcome in resectable parotid cancer, Eur J Surg Oncol 34, 932-937.

[4] R. Koul, A. Dubey, J. Butler et al., Prognostic factors depicting disease-specific survival in parotid-gland tumors, Int J Radiat Oncol Biol Phys 68, 714-718.

[5] L. Barnes, J. Eveson, P. Reichart et al., Pathology and Genetics of Head and Neck Tumours. World Health Organization Classification of Tumors, World Health Organization, 2005.

[6] V.V. Poorten, A. Hart, T. Vauterin et al., Prognostic index for patients with parotid carcinoma: international external validation in a Belgian-German database, Cancer 115, 540 550.

[7] M.F. Press, M.C. Pike, G. Hung et al., Amplification and overexpression of HER-2/neu in carcinomas of the salivary gland: correlation with poor prognosis, Cancer Res 54, 56755682 .
[8] A. Skalova, I. Starek, T. Vanecek et al., Expression of HER$2 /$ neu gene and protein in salivary duct carcinomas of parotid gland as revealed by fluorescence in-situ hybridization and immunohistochemistry, Histopathology 42, 348-356.

[9] J. Zhuang and H.J. Brady, Emerging role of Mcl-1 in actively counteracting BH3-only proteins in apoptosis, Cell Death Differ 13, 1263-1267.

[10] H. Schulze-Bergkamen, R. Ehrenberg, L. Hickmann et al., $\mathrm{Bcl}-\mathrm{x}(\mathrm{L})$ and Myeloid cell leukaemia-1 contribute to apoptosis resistance of colorectal cancer cells, World J Gastroenterol 14, 3829-3840.

[11] L.C. Whisler, N.B. Wood, D.D. Caldarelli et al., Regulators of proliferation and apoptosis in carcinoma of the larynx, Laryngoscope 108, 630-638.

[12] W. Sieghart, D. Losert, S. Strommer et al., Mcl-1 overexpression in hepatocellular carcinoma: a potential target for antisense therapy, J Hepatol 44, 151-157.

[13] W. Likui, L. Qun, Z. Wanqing et al., Prognostic role of myeloid cell leukemia-1 protein (Mcl-1) expression in human gastric cancer, J Surg Oncol 100, 396-400.

[14] C. Kempkensteffen, S. Hinz, M. Johannsen et al., Expression of Mcl-1 splicing variants in clear-cell renal cancer and their correlation with histopathological parameters and prognosis, Tumour Biol 30, 73-79.

[15] M. Genetzakis, I.P. Gomatos, A.N. Georgiou et al., BCL2, p53 and HLA-DR antigen expression in surgically treated parotid cancer patients, Eur Arch Otorhinolaryngol 266, 417424.

[16] S. Schneider, D. Thurnher, P. Kloimstein et al., Expression of the Sonic hedgehog pathway in squamous cell carcinoma of the skin and the mucosa of the head and neck, Head Neck.

[17] A.Y. Bedikian, M. Millward, H. Pehamberger, et al., Bcl-2 antisense (oblimersen sodium) plus dacarbazine in patients with advanced melanoma: the Oblimersen Melanoma Study Group, J Clin Oncol 24, 4738-4745.

[18] S. Trudel, Z.H. Li, J. Rauw et al., Preclinical studies of the pan$\mathrm{Bcl}$ inhibitor obatoclax (GX015-070) in multiple myeloma, Blood 109, 5430-5438.

[19] J.J. Shieh, K.T. Liu, S.W. Huang et al., Modification of alternative splicing of Mcl-1 pre-mRNA using antisense morpholino oligonucleotides induces apoptosis in basal cell carcinoma cells, J Invest Dermatol 129, 2497-2506.

[20] C. Thallinger, M.F. Wolschek, H. Maierhofer et al., Mcl-1 is a novel therapeutic target for human sarcoma: synergistic inhibition of human sarcoma xenotransplants by a combination of mcl-1 antisense oligonucleotides with low-dose cyclophosphamide, Clin Cancer Res 10, 4185-4191.

[21] C. Skoda, B.M. Erovic, V. Wachek et al., Down-regulation of Mcl-1 with antisense technology alters the effect of various cytotoxic agents used in treatment of squamous cell carcinoma of the head and neck, Oncol Rep 19, 1499-1503.

[22] H. Luukkaa, P. Klemi, I. Leivo et al., Prognostic significance of $\mathrm{Ki}-67$ and $\mathrm{p} 53$ as tumor markers in salivary gland malignancies in Finland: an evaluation of 212 cases, Acta Oncol 45, 669675.

[23] A. Sofiadis, E. Tani, T. Foukakis et al., Diagnostic and prognostic potential of MIB-1 proliferation index in thyroid fine needle aspiration biopsy, Int J Oncol 35, 369-374.

[24] H. Lee, T.J. Yoon, J.L. Figueiredo et al., Rapid detection and profiling of cancer cells in fine-needle aspirates, Proc Natl Acad Sci U S A 106, 12459-12464. 


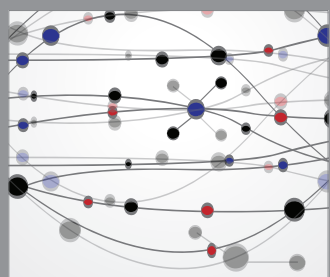

The Scientific World Journal
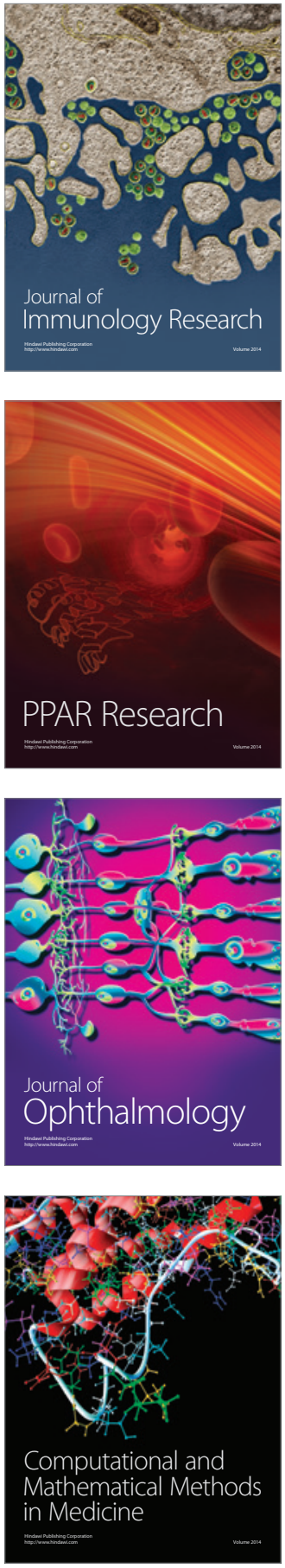

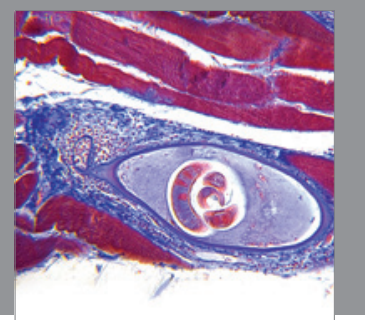

Gastroenterology

Research and Practice
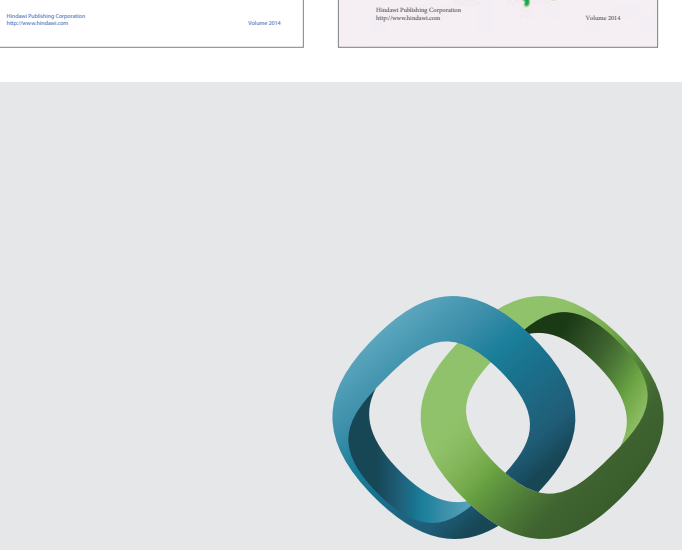

\section{Hindawi}

Submit your manuscripts at

http://www.hindawi.com
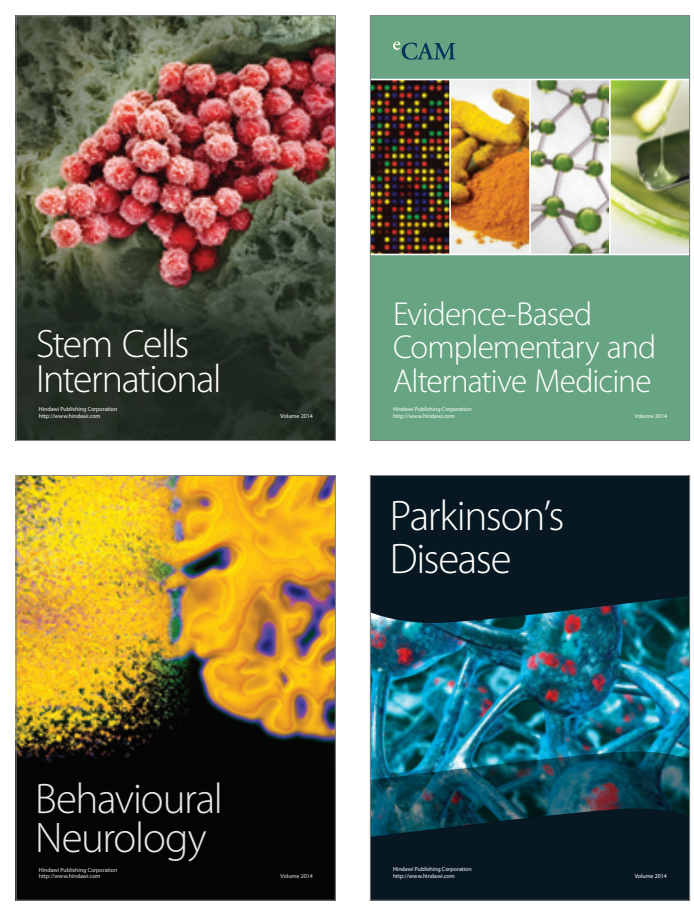

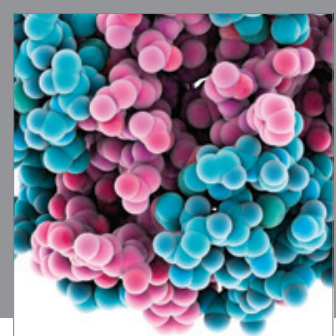

Journal of
Diabetes Research

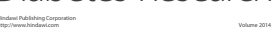

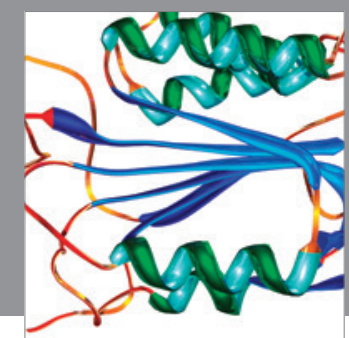

Disease Markers
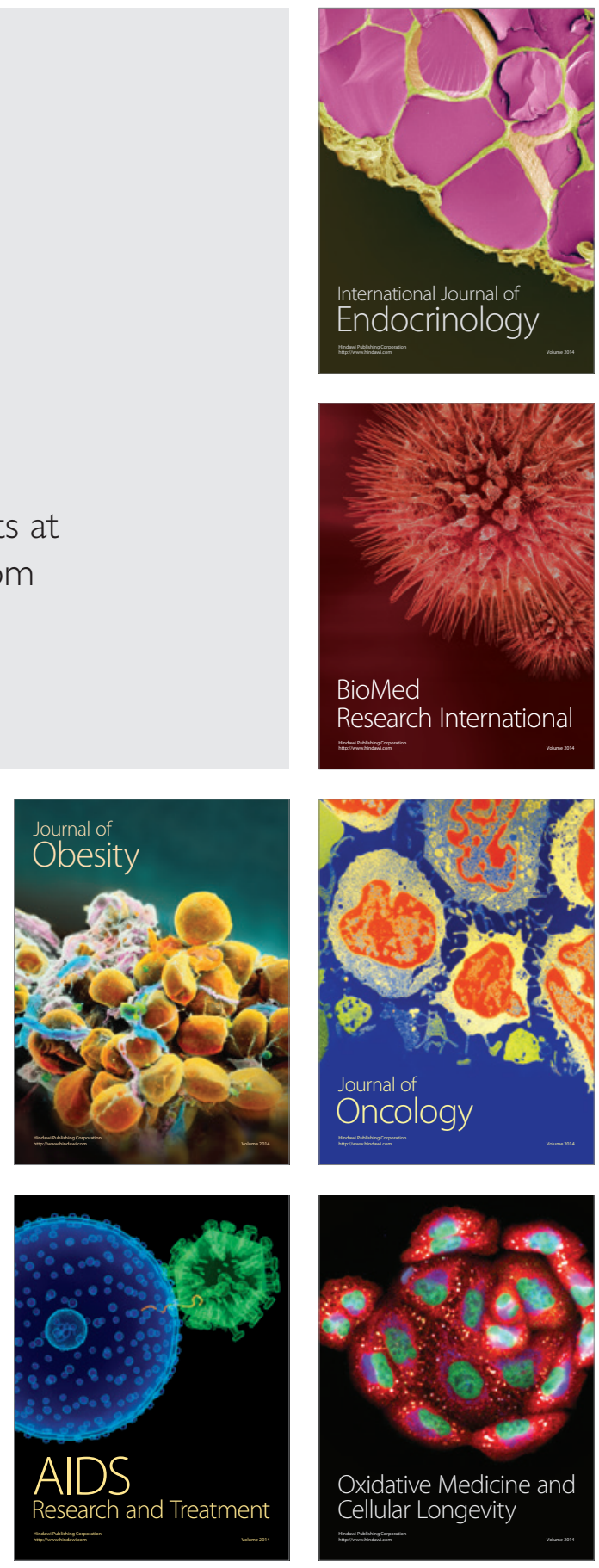